'instantons') generate $\mathrm{T}$ and $\mathrm{P}$ violating effects which involve a new parameter called $\theta$. Although $\theta$ could have any value, it would seem natural for it to be of order one. However, the experimental upper limit on the $T$ and $P$ violating electric dipole moment of the neutron requires $\theta<10^{-8}$. No good reason why $\theta$ should be so small is known.

Thus there are still some mysteries surrounding CP violation. Indeed, it is still not clear whether $C, P$ and $T$ are intrinsically broken. It is possible to construct electroweak theories in which the underlying equations are completely symmetrical but the solutions are asymmetrical. Recently interest in the nature of $\mathrm{CP}$ violation has been greatly increased by the discovery that it may be a key element in explaining the large excess of matter over antimatter in the Universe. Attempts to unify the strong with the electroweak interactions suggest that baryon number is not conserved. Indeed, they predict that the proton decays with a lifetime of about $10^{30}$ years, and experiments designed to search for proton decay are now under construction. In this case, as originally discussed by Sakharov, a universe containing mostly matter can develop from a state of equilibrium between matter and antimatter because of the asymmetry due to $\mathrm{CP}$ violation. Furthermore, this picture is capable of explaining the observed ratio of about $10^{8}$ between the number of photons and baryons in the Universe. If baryon number is conserved, it is necessary to invoke an initial asymmetry of one part in $10^{8}$ to explain this ratio. If these cosmological speculations hold up they will put severe constraints on the fundamental mechanism responsible for $\mathrm{CP}$ violation.

The discovery of $\mathrm{CP}$ violation completed the conceptual revolution begun by the discovery of parity violation. This revolution has led to a healthy scepticism about other old shibboleths such as baryon conservation. Current theory can naturally accommodate $\mathrm{CP}$ violation. It is true that its magnitude cannot be predicted at present but this problem is on the same footing as other unanswered questions, such as how to predict the masses of quarks, if we leave aside the $\theta$ problem. The asymmetry between matter and antimatter which previously seemed "more than strange" now seems natural and perhaps even inevitable. But without it the Universe might be quite different.

\title{
Genetic engineering and foot and mouth disease vaccines
}

\section{from J. B. Brooksby}

WHEN genetic engineering was first discussed in relation to foot and mouth disease vaccines, conventional virologists were sceptical of a practical outcome in less than perhaps ten or fifteen years. Enough was known of the viral genome to suggest that appropriate information might be incorporated into plasmids but a pessimistic view was taken of the possibility of expression of this information to provide the antigenic moiety of the virus in active form. The paper by Kupper and his co-authors on p. 555 of this issue shows that the obstacle of expression has been surmounted and a milestone has been passed on the road to production of antigens for vaccine.

Although this is not the first report of the production of a viral antigen in this way, it is a happy circumstance that a foot and mouth disease product is early in the field, not perhaps because the agent was the first animal pathogen discovered to be a virus, but because of the importance of foot and mouth disease vaccine in controlling what is still one of the most serious diseases of animals throughout the world. More foot and mouth disease vaccine is produced than any other vaccine, but more is yet needed to begin campaigns in countries where the disease continues to spread. Compulsory regular vaccination in Western European countries over the past 25 years covering more than 60 million cattle has reduced the incidence of the disease to vanishing point - indeed, most recent outbreaks have been linked, at least circumstantially, to vaccine containing residual live virus. In South America, which has a long-standing problem, at

J. B. Brooksby is a former Director of the Animal Virus Research Centre, Pirbright, $U K$. least 500 million doses of polyvalent vaccine are produced annually. These figures indicate the importance of the disease to the animal industry, through its debilitating effects, lowering of milk production and interference, because of its remarkable spreading power, with trade in animals, meat and other products.

Present vaccines are produced from virus cultivated either in surviving tongue epithelial tissue collected from animals slaughtered for meat, or in cell lines grown in suspension in large $(2,000$ litres or more) vessels. Problems arise in the production of sufficient viral antigen, especially with some strains of virus, and the multiplicity of types (there are seven) and sub-types (over 60) necessitates either expensive polyvalent vaccines or vaccines appropriate to particular areas which may have to be changed rapidly to meet new strains arising in the field. Thus, although much has been achieved with available vaccines, as the experience of European countries shows, there is still a real need for alternative methods for producing viral antigen.

The application of genetic engineering may offer such a method and the work reported, together with other studies proceeding in the UK (at the Animal Virus Research Institute and Wellcome Foundation Ltd) and in the US (at the Plum Island Animal Disease Center and Genentech - see Nature 288, 663; 1979), indeed give promise for the future.

The advantages of a vaccine based on an antigen consisting of the appropriate viral protein, prepared in a way that does not involve infective material at any subsequent stage after isolation of the viral RNA and which allows the production of greater quantities than from natural sources, are obvious. Kupper et al. have concentrated on the preparation of VP1, the coat protein responsible for the production of the antibody response to foot and mouth disease virus. Laporte and Bachrach and their colleagues in France and the US showed that purified VP1 from disrupted virus stimulates an antibody response, albeit weak, in guinea pigs and swine, and large doses will protect swine against subsequent infection.

In the present experiments, VP1 produced in the bacterial cell has been identified by its reaction with specific VP1 antibody and from the serological reaction it is estimated that at least 1,000 VP1 molecules are synthesized per cell. This is a signal achievement which is not diminished by the question - what remains to be done? Clearly, the new VP1 must be shown to immunize animals. The quantitative aspect is important, for the earlier work suggested that at least 1,000 times more antigen was required when the VP1 had been isolated than when it was carried on the intact, but inactivated virus particle. Yields from bacterial culture can probably be raised above those from mammalian cell culture and the factor by which this is possible will be critical for the success of the method. The quality of the antigen is also fundamental. It may be that, in order to preserve VP1 from degradation, it will be advantageous to synthesize the precursor which cleaves to form all four capsid proteins.

A new and exciting prospect is the possibility of overcoming some of the difficulties created by virus variation in the field. It may well be practicable to tailor strains to stimulate immunity to a wider antigenic spectrum than that given by naturally occurring viruses. There is no doubt that the progress reported will lead to a redoubled effort by the various groups and the prospects for new foot and mouth disease vaccines are indeed bright. 